\title{
Design and fabrication of anti-reflection coating on Gallium Phosphide, Zinc Selenide and Zinc Sulfide substrates for visible and infrared application
}

\author{
D. Vápenka ${ }^{1,2, a}$, J. Václavík ${ }^{1}$, and P. Mokrý1,2 \\ ${ }^{1}$ Research Centre for Special Optics and Optoelectronic Systems (TOPTEC), Institute of Plasma Physics, Academy of Sciences \\ of the Czech Republic, Sobotecká 1660, Turnov, CZ-51101, Czech Republic \\ ${ }^{2}$ Institute of Mechatronics and Technical Engineering, Technical University of Liberec, Studentská 2, Liberec, CZ-46117, Czech \\ Republic
}

\begin{abstract}
Results of design and fabrication of a dual-band anti-reflection coating on a gallium phosphide $(\mathrm{GaP})$, zinc selenide $(\mathrm{ZnSe})$ and zinc sulfide $(\mathrm{ZnS})$ substrates are presented. A multilayer stack structure of antireflection coatings made of zinc sulfide and yttrium fluoride (YF3) was theoretically designed for optical bands between 0.8 and $0.9 \mu \mathrm{m}$ and between 9.5 and $10.5 \mu \mathrm{m}$. This stack was designed as efficient for these materials ( $\mathrm{GaP}, \mathrm{ZnS}, \mathrm{ZnSe})$ together. Multilayer stack structure was deposited using thermal evaporation method. Theoretically predicted transmittance spectra were compared with transmitted spectra measured on coated substrates. Efficiency of anti-reflection coating is estimated and discrepancies are analyzed and discussed.
\end{abstract}

\section{Introduction}

Nowadays, grinding and polishing techniques for various optical materials applicable for visible (VIS) and infrared (IR) range applications are the subject of an intense applied research. Some of materials, which is now frequently used in VIS and IR applications, are gallium phosphide $(\mathrm{GaP})$, zinc selenide ( $\mathrm{ZnSe}$ ) and zinc sulfide ( $\mathrm{ZnS})$. Transmittance of these materials is in the range $55-70 \%$ in the optical region from $550 \mathrm{~nm}$ to $12 \mu \mathrm{m}$ [1] due to high refractive indices and associated reflections on surfaces. It is possible to increase the transmittance (i.e. decrease the reflectance) of these samples by proper anti-reflective coatings. Suitable materials for the use in these samples coatings should be chosen with a particular consideration of their thermal evaporation and their bandwidth. The optimal design, i.e. thicknesses of particular layers in the multilayer coating, should be computed with an additional consideration of possible inaccuracies that may occur in the deposition process.

To obtain desired wavelength dependence of the transmittance for samples of different materials, it is necessary to create different thin-layer structure for each material. Even in the case, that the desired transmittance is the same. However, sometimes it is possible to create compromise design, which is sufficient for all these materials. Thus it can increase efficiency of coating process during piece production of optical system which includes optical elements made from these materials.

Objective of the work presented in this paper was a deposition of an anti-reflective coating on a $\mathrm{GaP}, \mathrm{ZnS}$ and $\mathrm{ZnSe}$ substrates. The maximum value of transmittance

\footnotetext{
a e-mail: vapenka@ipp.cas.cz
}

was required to be between 800 and $900 \mathrm{~nm}$ in VIS optical band and between 9.5 and $10.5 \mu \mathrm{m}$ in IR optical band for all these materials. Wavelengths between 800 and 900 $\mathrm{nm}$ are not visible by human eye, but they are detectable by the silicon detectors that are used for imaging in visible range. Therefore our criterium for designating it as VIS range is detectability by Si detectors. The optimal design was computed in the OptiLayer software. The emphasis was put on a simplicity, robustness of the design and on usability to cover all these materials simultaneously.

\section{Design of anti-reflection coating}

The standard structure of a thin film multilayer structure consists of alternating layers of two materials. One material should have a high refractive index $(\mathrm{H})$ and the other material low (L). Reflectivity of the multilayer coating is essentially controlled by the choice of materials and the thicknesses of layers. As it was mentioned above, materials will be deposited by thermal evaporators. Thus, it is important to use materials suitable for a thermal evaporation. Furthermore, the materials must have a low absorption between 0.8 and $10.5 \mu \mathrm{m}$.

In accordance with the aforementioned restrictions, following materials were chosen: $\mathrm{ZnS}$ as an $\mathrm{H}$ material, and YF3 as an L material. Transparency of ZnS and YF3 cover the range from 0.4 to $14 \mu \mathrm{m}$ and the range from 0.3 to $12.5 \mu \mathrm{m}$, respectively [1][2]. Both materials can be deposited by thermal evaporation. In Figure 1, one can see the designed transmittance plotted as a function of wavelength (computed using the OptiLayer software). It can be seen that position of transmittance peak varies for each material. For $\mathrm{ZnS}$ and $\mathrm{ZnSe}$ is peak position very close.

This is an Open Access article distributed under the terms of the Creative Commons Attribution License 2.0, which permits unrestricted use, distribution, and reproduction in any medium, provided the original work is properly cited. 


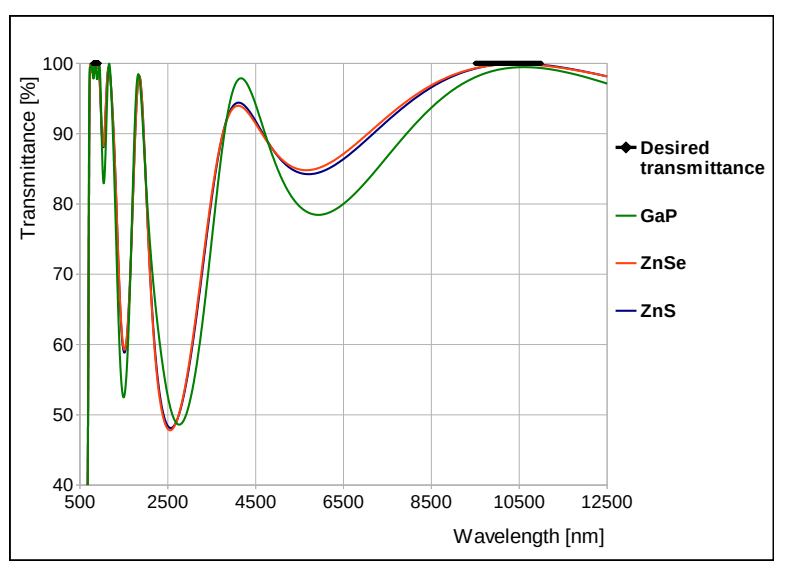

Figure 1. Dependence of designed transmittance as a function of wavelength.

For GaP is maximum of transmittance slightly shifted toward longer wavelengths. However, in this case is this shift insignificant. The design is relatively insensitive to the thickness variation. Thus, a small change in the thickness, e.g. up to $5 \%$, has a minimum effect on the transmittance peak position in the desired wavelength. Table 1 lists layers that should be evaporated, in order to obtain the designed transmittance dependence on wavelength. One can see in table, that the first layer is from $\mathrm{H}$ material $(\mathrm{ZnS})$. It is quite important for improving the sensitivity of photometric measurement of thickness. As will be discussed in the next chapter. Despite the relative simplicity of the designed multilayer structure (as it has only 6 layers), the efficiency of the anti-reflective coating should be quite acceptable (with respect to the design).

Table 1. Designed recipe of layers (the thicknesses are in $\mathrm{nm}$ )

\begin{tabular}{|l|l|l|l|}
\hline & Phys. thickness & Opt. thickness & Material \\
\hline \hline 1. & 275.17 & 619.15 & $\mathrm{H}$ \\
\hline 2. & 309.28 & 462.44 & $\mathrm{~L}$ \\
\hline 3. & 210.36 & 473.32 & $\mathrm{H}$ \\
\hline 4. & 577.57 & 863.53 & $\mathrm{~L}$ \\
\hline 5. & 211.38 & 475.60 & $\mathrm{H}$ \\
\hline 6. & 169.59 & 253.56 & $\mathrm{~L}$ \\
\hline
\end{tabular}

\section{Experimental procedure}

Deposition of the multilayer coating was done in a vacuum using an evaporation from thermal sources. Table 2 lists the deposition parameters. Deposition rate and thicknesses

Table 2. Deposition parameters

\begin{tabular}{|l|l|}
\hline Parameter & Value \\
\hline \hline ZnS deposition rate & $10 \AA / \mathrm{s}$ \\
\hline YF3 deposition rate & $5 \AA / \mathrm{s}$ \\
\hline Substrate temperature & $165-180^{\circ} \mathrm{C}$ \\
\hline Pressure & $8 \times 10^{-4}-1 \times 10^{-3} \mathrm{~Pa}$ \\
\hline
\end{tabular}

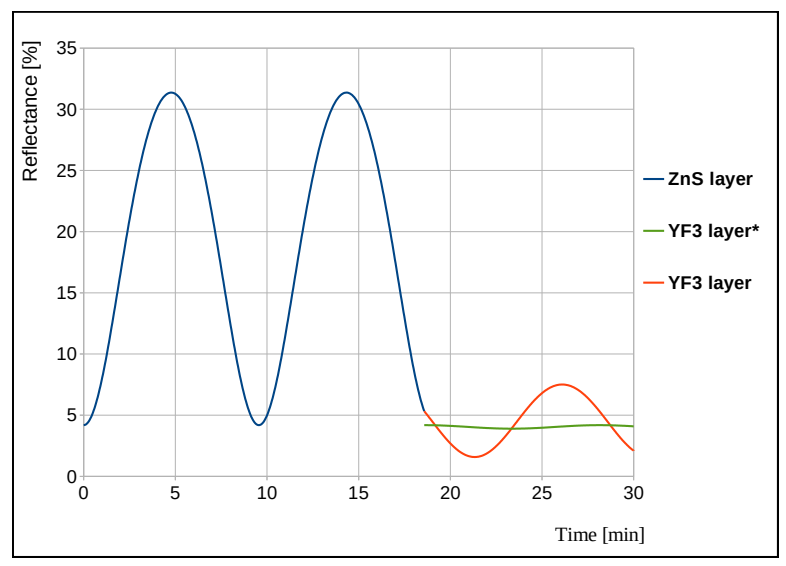

Figure 2. Comparison of signal strength during reflectivity measurement. The red line represents time dependence of reflectivity of YF3 layer during evaporation onto the measuring glass with $\mathrm{ZnS}$ inter-layer. The green line represents coating of YF3 onto clean surface of the measuring glass.

of the layers was measured by a quartz monitor (QCM). Termination of each layer was performed with the use of a reflection photometer. As mentioned above, the first layer is made of $\mathrm{ZnS}$. Reason is the low change in reflectivity during deposition YF3 onto a measuring glass (refractive indices of YF3 and the measuring glass are relatively similar). If the first layer is made of YF3, photometer sensitivity i.e. variation of signal with optical thickness, would be very low which would cause an thickness errors. The comparison of signal strength during reflectivity measurement of YF3 on the measuring glass with and without $\mathrm{ZnS}$ inter-layer, is shown in Figure 2.

The combination of materials that have been used for evaporation, is in terms of substrate temperature slightly problematic. Optimal substrate temperature for $\mathrm{ZnS}$ coatings is lower $\left(150-175^{\circ} \mathrm{C}\right)$ than for $\mathrm{YF} 3$ coatings $(200-$ $250^{\circ}$ )[3][4]. Therefore the substrate temperature was chosen as a compromise $\left(165-180^{\circ}\right)$. An increase in a substrate temperature occurred during the deposition. Thus, the evaporation of the layers 1 and 4 was intermittent. When the temperature was undesirably high, the ongoing evaporation process was paused until the temperature has decreased. After that, the evaporation process was resumed.

\section{Results and discussion}

Transmittance of the coated substrates was measured after its deposition. In Figures 3, 4 and 5, one can see results from a VIS spectrometer. First figure compares transmittance of uncoated samples with samples which have been coated from one side. The second figure compares uncoated and both side coated samples. The third figure shows difference in transmittance between uncoated and both side coated samples. All of these figures shows good agreement with the theoretical design. The peaks of transmittance are located in desired ranges of wavelengths and they aren't almost shifted with each other. With regard 


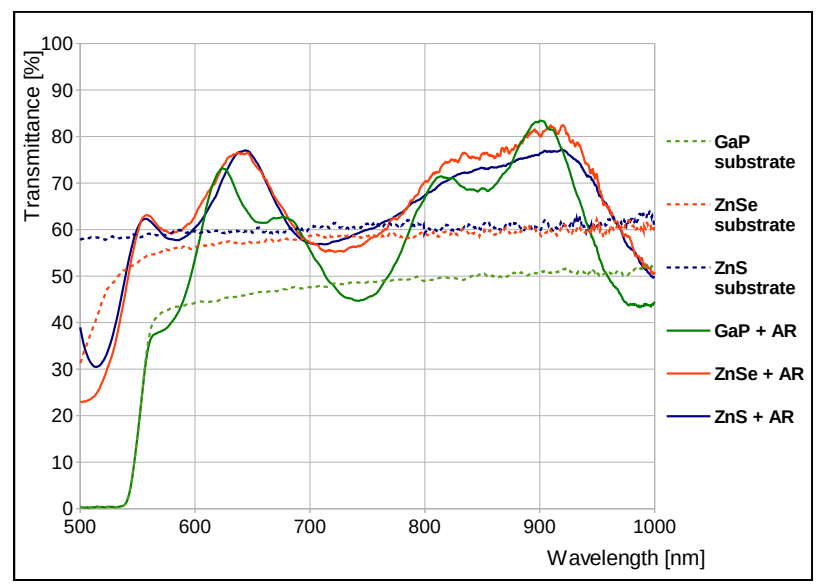

Figure 3. Wavelength dependence of the transmittance of GaP, $\mathrm{ZnS}$ and $\mathrm{ZnSe}$ substrates with and without anti-reflective coating (on the one side) in VIS optical range.

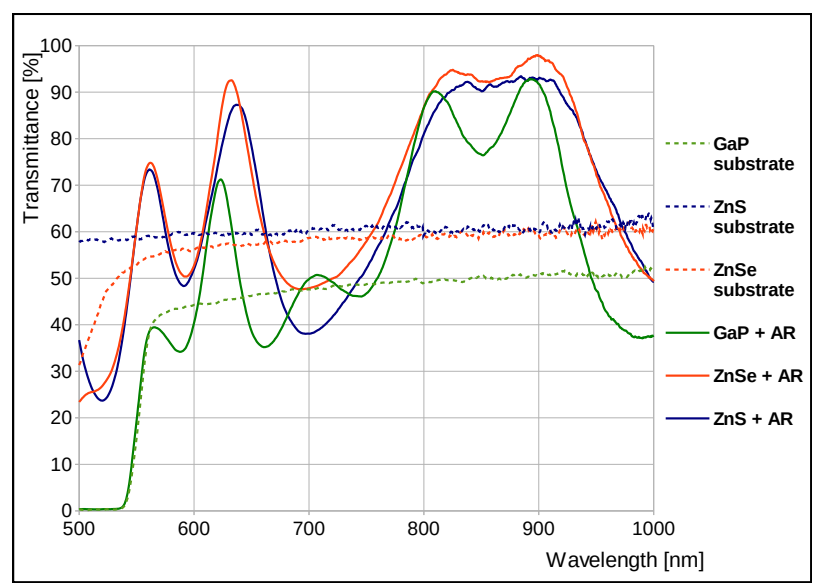

Figure 4. Wavelength dependence of the transmittance of GaP $\mathrm{ZnS}$ and $\mathrm{ZnSe}$ substrates with and without anti-reflective coating (on both sides) in VIS optical range.

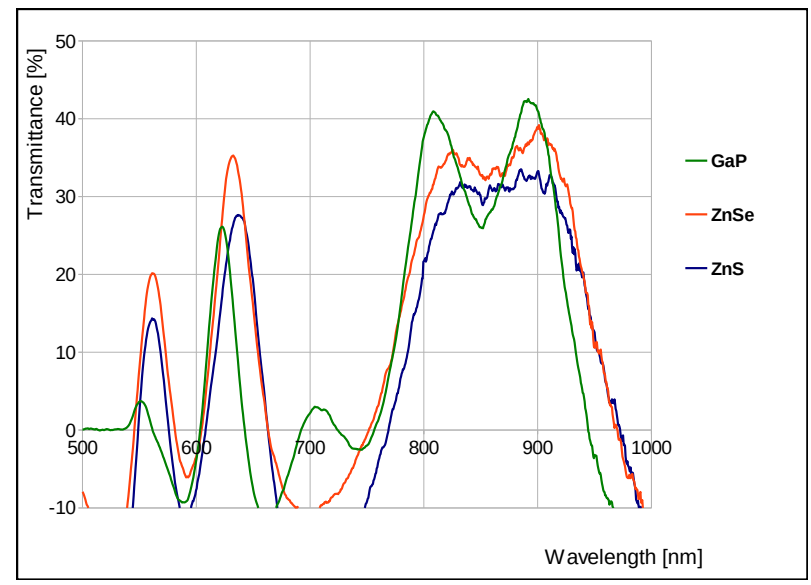

Figure 5. Wavelength dependence of the transmittance difference between uncoated and both side coated $\mathrm{GaP}, \mathrm{ZnS}$ and $\mathrm{ZnSe}$ substrates in VIS optical range.

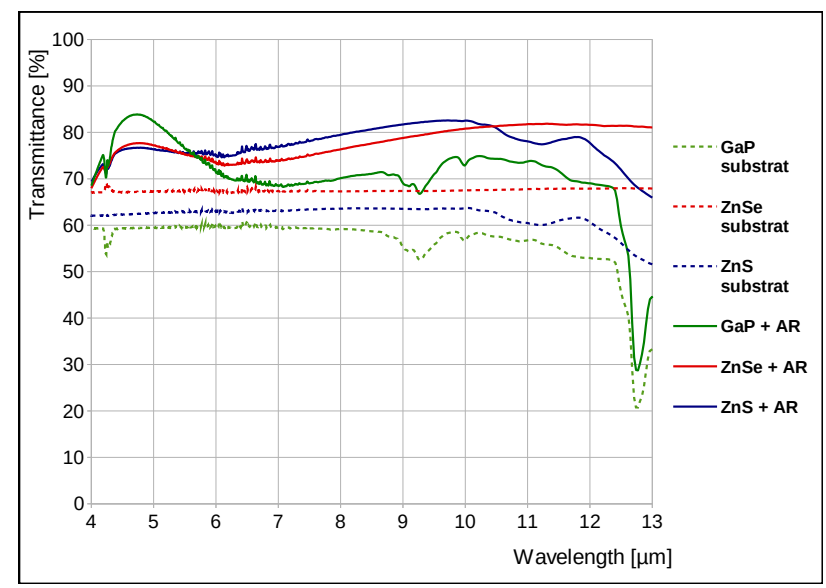

Figure 6. Wavelength dependence of the transmittance of GaP, $\mathrm{ZnS}$ and $\mathrm{ZnSe}$ substrates with and without anti-reflective coating (on the one side) in IR optical range.

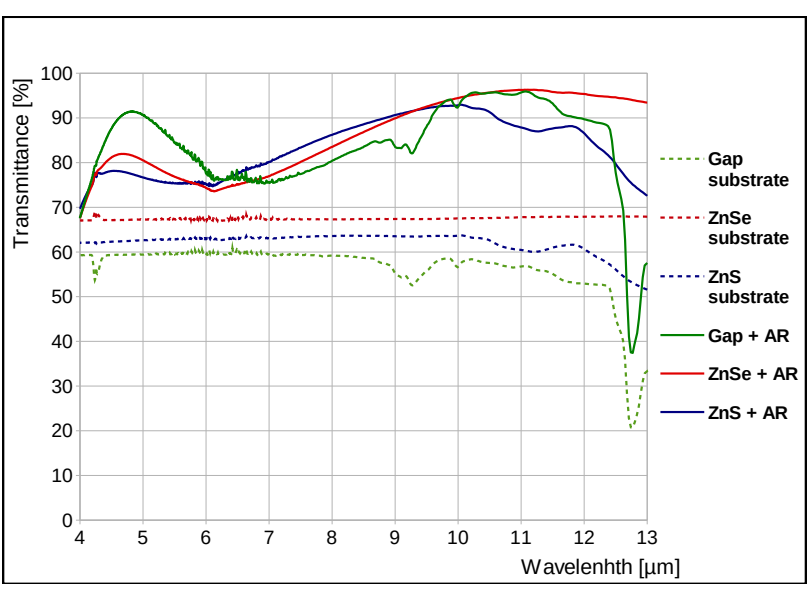

Figure 7. Wavelength dependence of the transmittance of GaP, $\mathrm{ZnS}$ and $\mathrm{ZnSe}$ substrates with and without anti-reflective coating (on both side) in IR optical range

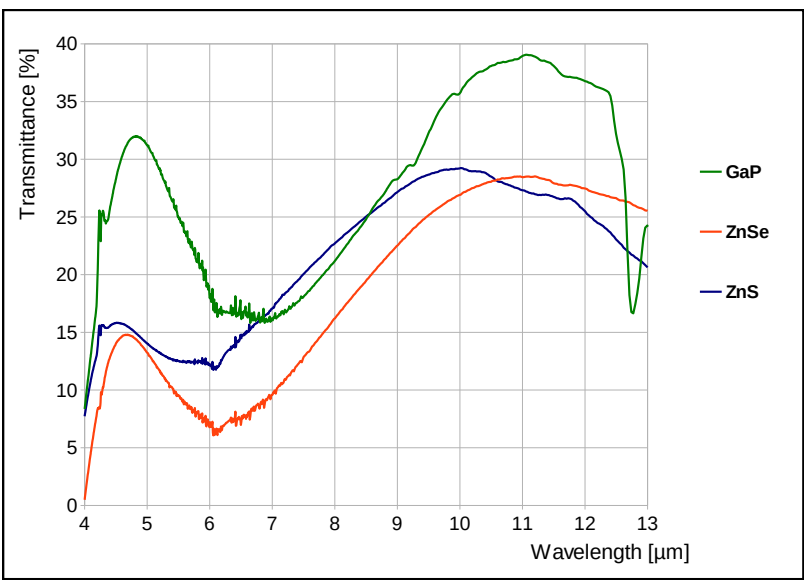

Figure 8. Wavelength dependence of the transmittance difference between uncoated and both side coated $\mathrm{GaP}, \mathrm{ZnS}$ and $\mathrm{ZnSe}$ substrates in IR optical range. 
to values of transmittance in selected wavelength range, an increase by approximately $40 \%$ is clearly seen in the optical range above $900 \mathrm{~nm}$.

The second measurement was performed using an IR spectrometer. Figures 6,7 and 8 shows the wavelength dependence of transmittance in the IR range. It is evident, that the anti-reflective coating increases the values of GaP transmittance by approximately $40 \%$ in the range around $11 \mu \mathrm{m}(\mathrm{ZnS}$ and $\mathrm{ZnSe}$ by almost $30 \%)$. It is good increase. With regard to the positions of peaks of transmittance, it is in reasonably good agreement with the design. $\mathrm{ZnS}$ is slightly shifted towards shorter wavelengths, $\mathrm{ZnSe}$ is shifted in the oposite direction and maximum transmittance of GaP sample is between them. However, according to the design, the peaks positions of $\mathrm{ZnS}$ and $\mathrm{ZnSe}$ transmittance should be closer to each other. This discrepancy could be caused by an angle error during mounting of the samples into the evaporation chamber. This error might cause a little bit different thicknesses of layers ( between the samples).

Although the measured absolute values do not agree with the theoretical design, the transmittance peak position and value well agree with the theoretical prediction. In Table 3, there is a comparison between designed and evaporated thicknesses of layers. The evaporated thicknesses (on the measuring glass sample) were measured by an ellipsometer. It shows that differences are relatively small (the largest difference is 5\%). Therefore, the evaporation process was successful.

Table 3. Comparison between designed and deposited thicknesses

\begin{tabular}{|l|l|l|}
\hline & Designed thickness & Deposited thickness \\
\hline \hline 1. & 275.17 & 280.2 \\
\hline 2. & 309.28 & 305.6 \\
\hline 3. & 210.36 & 213.5 \\
\hline 4. & 577.57 & 584.3 \\
\hline 5. & 211.38 & 215.3 \\
\hline 6. & 169.59 & 176.3 \\
\hline
\end{tabular}

\section{Conclusion}

The anti-reflection coating made of six alternating $\mathrm{ZnS}$ and YF3 layers has been deposited on a GaP, $\mathrm{ZnS}$ and $\mathrm{ZnSe}$ substrates using an evaporation from thermal sources. Measurement on a VIS and IR spectrometer confirmed a good improvement in the transmittance in the both regions (by up to $40 \%$ ).

We managed to create a design that is reasonably applicable to substrates of three different materials $(\mathrm{ZnS}$, $\mathrm{ZnSe}, \mathrm{GaP})$ which have significantly different refractive indices. These materials are used for elements of highperformance, wide-bandwidth optical systems which are not produced in large series. This design could potentially increase the efficiency of coating process during production of the optical system.

It can be concluded that the theoretical results predicted during the design of the optimal anti-reflection coating reasonably agree with data obtained from experiments.

\section{Acknowledgements}

This work was supported by the Ministry of Education, Youth and Sports of the Czech Republic in the Project EUREKA E!3869 Infrasens OE10003, by the European Regional Development Fund and the Ministry of Education, Youth and Sports of the Czech Republic in the Project No. CZ.1.05/2.1.00/03.0079: Research Center for Special Optics and Optoelectronic Systems (TOPTEC), by the Student Grant SGS 2012/7821 Interactive Mechatronics Systems Using the Cybernetics Principles.

\section{References}

[1] Daniel C.Harris, Infrared Physics \& Technology 39, 185-201 (1998)

[2] H. K. Pulker, Applied Optics, 18, 1969-1977 (1979)

[3] Materion Corporation, Yttrium Fluoride YF3 Materion, (2013)

[4] Materion Corporation, Cirom -2 zinc Sulfide Materion, (2013) 